Original Research Paper

\title{
A Note on Improved Young Type Inequalities with Kantorovich Constant
}

\author{
Leila Nasiri and Mahmood Shakoori \\ Department of Mathematics, Lorestan University, Khorramabad, Iran
}

\author{
Article history \\ Received: 20-04-2016 \\ Revised: 14-09-2016 \\ Accepted: 16-09-2016 \\ Corresponding Author: \\ Leila Nasiri \\ Department of Mathematics, \\ Lorestan University, \\ Khorramabad, Iran \\ Email: leilanasiri468@gmail.com
}

\section{Introduction}

The scalar Young inequality says that if $a$ and $b$ be nonnegative real numbers and $0 \leq v \leq 1$, then:

$$
a^{v} b^{1-v} \leq v a+(1-v) b
$$

With equality if and only if $a=b$ : Inequality (1.1) is called the $v$-weighted arithmetic-geometric mean inequality. If $v=\frac{1}{2}$ then:

$$
\sqrt{a b} \leq \frac{a+b}{2}
$$

Which is called arithmetic-geometric mean.

Zuo et al. (2011) refined Young inequality (1.1) as follows:

$$
v a+(1-v) b \geq K(h, 2)^{\mathrm{r}} a^{v} b^{1-v}
$$

For all $a, b \geq 0$ and $v \in[0,1]$, where $r=\min \{v, 1$ $-v\}, h=\frac{a}{b}$ and $K(h, 2)=\frac{(h=2)^{2}}{4 h}$, so that $K(h, 2)$ is called Kantorovich constant.

Here, we need to recall that Kantorovich constant satisfies the following properties:

(i) $K(1,2)=1$,

(ii) $K(t, 2)=K\left(\frac{1}{t} 2\right)$

(iii) $K(t, 2)$ is monotone increasing on $[1, \infty)$ and is monotone decreasing on $(0,1]$
Wu and Zhao (2013) improved inequality (1.3) in the following form:

$$
v_{a}+(1-v) b \geq \Upsilon(\sqrt{a}-\sqrt{b})^{2}+K(\sqrt{h}, 2)^{\Upsilon^{\prime}} a^{v} b^{1-v}
$$

For all $a, b \geq 0$ and $v \in[0,1]$; where $h=\frac{a}{b}, r=\min$ $\{v, 1-v\}$ and $r^{\prime}=\min \{2 r, 1-2 r\}$.

By (Nasiri et al., 2016) we give some improved inequalities of (1.1) as follows:

$$
\begin{aligned}
v^{2} a^{2}+(1-v)^{2} b^{2} & \geq(v a)^{2 v} b^{2-2 v}+v^{2}(a-b)^{2} \\
+ & r_{0} b(\sqrt{v a}-\sqrt{b})^{2}, 0 \leq v \leq \frac{1}{2}
\end{aligned}
$$

where, $h=\frac{v a}{b}$ and $r=\min \{2 v, 1-2 v\}$.

And:

$$
\begin{gathered}
v^{2} a^{2}+(1-v)^{2} b^{2} \geq a^{2 v}[(1-v) b]^{2-2 v}+(1-v)^{2}(a-b)^{2} \\
+r_{0} a(\sqrt{a}-\sqrt{(1-v) b})^{2}, \frac{1}{2} \leq v \leq 1
\end{gathered}
$$

where, $h=\frac{a}{(1-v) b}$ and $r_{0}=\min \{2 v-1,2(1-v)\}$.

Suppose $M_{n}$ be the space of $n \times n$ complex matrices. Suppose $\|$.$\| denote any unitarily invariant norm on M_{n}$. So, $\|U A V\|=\|A\|$ for all $A \in M_{n}$ and for all unitary matrices $U, V \in M_{n}$. For $A=\left[a_{i j}\right] \in M_{n}$, the HilbertSchmidt norm (or Frobenious) and the trace norm of $A$ are defined by: 


$$
\|A\|_{2}=\sqrt{\sum_{j=1}^{n} S_{j}^{2}(A)}\|A\|_{1}=\sum_{j=1}^{n} S_{j}(A)
$$

Respectively, where $s_{1}(A) \geq s_{2}(A) \geq \ldots \ldots . \geq s_{n}(A)$ are the singular values of $A$, that is, the eigenvalues of the positive matrix $|A|=\left(A^{*} A\right)^{\frac{1}{2}}$ arranged in decreasing order and repeated according to multiplicity. It is known that the Hilbert-Schmidt norm is unitarily invariant.

For more information about Young type inequality and its matrix version the reader is referred to (Bhatia, 1996; Hu, 2012).

\section{Inequalities for Scalars}

In this section, we will derive some Young type inequalities for scalars.

Theorem 2.1. Let $a, b \geq 0$ and $0 \leq v \leq 1$.

(i) If $0 \leq v \leq \frac{1}{2}$, then:

$$
\begin{aligned}
& v^{2} a^{2}+(1-v)^{2} b^{2} \geq v^{2}(a-b)^{2} \\
& +r b(\sqrt{b}-\sqrt{v a})^{2}+K(\sqrt{h v}, 2)^{r^{\prime}}\left[(v a) v b^{1-v}\right]^{2}
\end{aligned}
$$

where, $h=\frac{a}{b}, r=\min \{2 v, 1-2 v\}$ and $r^{\prime}=\min \{2 r, 1-2 r\}$.

(ii) If $\frac{1}{2} \leq v \leq 1$, then

$v^{2} a^{2}+(1-v)^{2} b^{2} \geq(1-v)^{2}(a-b)^{2}+r a(\sqrt{a}-\sqrt{(1-v) b})^{2}$

$+K\left(\sqrt{\frac{h}{1-v}}, 2\right)^{r^{\prime}}\left[a^{v}((1-v) b)^{1-v}\right]^{2} a^{2 v}$

where, $h=\frac{a}{b}, r=\min \{2 v-1,2(1-v)\}$ and $r=\min \{2 r$, $1-2 r\}$.

Proof: Let $0 \leq v \leq \frac{1}{2}$. Then we have:

$$
\begin{aligned}
& v^{2} a^{2}+(1-v)^{2} b^{2}-v^{2}(a-b)^{2} \\
& =b[(1-2 v) b+2 v(v a)] \\
& \geq b\left[r(\sqrt{b}-\sqrt{v a})^{2}+K(\sqrt{v h}, 2) r^{\prime} b^{1-2 v}(v a)^{2 v}\right] \\
& =r b(\sqrt{b-\sqrt{v a}})^{2}+K(\sqrt{v h}, 2) r^{\prime} b^{2-2 v}(v a)^{2 v}
\end{aligned}
$$

That is:

$$
\begin{aligned}
v^{2} a^{2} & +(1-v)^{2} b^{2} \geq v^{2}(a-b)^{2}+r b(\sqrt{b}-\sqrt{v a})^{2} \\
& +K(\sqrt{h v}, 2) r^{\prime}\left[(v a)^{v} b^{1-v}\right]^{2}
\end{aligned}
$$

Thus, (2.1) holds.

For $\frac{1}{2} \leq v \leq 1$, compute:

$$
\begin{aligned}
& v^{2} a^{2}+(1-v)^{2} b^{2}-(1-v)^{2}(a-b)^{2} \\
& =a[(2 v-1) a+2(1-v)((1-v) b)] \\
& \geq a\left[\begin{array}{l}
r(\sqrt{a}-\sqrt{(1-v) b})^{2} \\
+K\left(\sqrt{\frac{h}{1-v}}, 2\right) r^{\prime} a^{2 v-1}\left(b(1-v)^{2(1-v)}\right.
\end{array}\right] \\
& =r b(\sqrt{a}-\sqrt{(1-v) b})^{2}+K\left(\sqrt{\frac{h}{1-v}}, 2\right) r^{\prime}\left[a^{v}(b(1-v))^{1-v}\right]^{2}
\end{aligned}
$$

Hence:

$$
\begin{aligned}
& v^{2} a^{2}+(1-v)^{2} b^{2} \geq(1-v)^{2}(a-b)^{2}+r a(\sqrt{a}-\sqrt{(1-v) b})^{2} \\
& K\left(\sqrt{\frac{h}{1-v}}, 2\right) r^{\prime}\left[a^{v}\left(b(1-v)^{1-v}\right]^{2}\right.
\end{aligned}
$$

This estimate completes the proof of (2.2); Theorem 2.1 is thereby proved.

Remark 1: Clearly, inequalities (2.1) and (2.2) are improvement of inequalities (1.5) and (1.6).

\section{Inequalities for Matrices}

According to the results obtaining from section 2, we present the trace and the Hilbert-Schmidt norm versions.

Let $A, B, X \in M_{n}$ so that $A$ and $B$ are positive semidefinite. We recall since $A, B>0$, thus $A=U D U^{*}$ and $B=V E V^{*}$ where $D=\operatorname{diag}\left(\lambda_{1}, \ldots, \lambda_{n}\right)$ and $E=$ $\operatorname{diag}\left(\mu_{1}, \ldots, \mu_{n}\right), \lambda i, v_{i} \geq 0$ for $1 \leq i \leq n$. Moreover, we mention that $\lambda_{i}$ and $\mu_{i}$ are the singular values of $A$ and $B$ (respectively). It is showed (Nasiri et al., 2016) that:

$$
\begin{aligned}
& \| v A X+\left(1-v X B\left\|_{2}^{2}+v^{2 v}\right\| A^{v} X B^{1-v}\left\|_{2}^{2}+v^{2}\right\|\right. \\
& A X-X B \|_{2}^{2}+r_{0}\left[v\left\|A^{\frac{1}{2}} X B^{\frac{1}{2}}\right\|_{2}^{2}+\right. \\
& \left.\|X B\|_{2}^{2}-2 \sqrt{v}\left\|A^{\frac{1}{4}} X B^{\frac{3}{4}}\right\|_{2}^{2}\right]+2 v(1-v)\left\|A^{\frac{1}{2}} X B^{\frac{1}{2}}\right\|_{2}^{2}
\end{aligned}
$$

where,

$$
K=\min \left\{\left(k \frac{v \lambda_{i}}{\mu_{j}}, 2\right) i, j=1, \ldots ., n\right\}
$$

$$
r=\min \{2 v, 2 v-1\}:
$$

$$
\begin{gathered}
\| v A X+\left(1-v X B\left\|_{2}^{2} \geq(1-v)^{2-2 v}\right\| A^{v} X B^{1-v}\right. \\
\left\|_{2}^{2}+(1-v)^{2}\right\| A X-X B \|_{2}^{2}+ \\
r_{0}\left[(1-v)\left\|A^{\frac{1}{4}} X B^{\frac{1}{2}}\right\|_{2}^{2}+\|A X\|_{2}^{2}-2 \sqrt{1-v} \| A^{\frac{3}{4}} X B^{\frac{1}{4}}\right. \\
\left.\|_{2}^{2}\right]+2 v(1-v)\left\|A^{\frac{1}{2}} X B^{\frac{1}{2}}\right\|_{2}^{2}
\end{gathered}
$$


where, $\quad K=\min \left\{\left(k \frac{\lambda_{i}}{(1-v) \mu_{j}}, 2\right) i, j=1, \ldots ., n\right\} \quad$ and $r=\min \{2 v-1,2-2 v\}$.

Our first main result is the following.

Theorem 3.1. Let $A, B, X \in M_{n}$ so that $A$ and $B$ are positive semidefinite.

(i) If $0 \leq v \leq \frac{1}{2}$, then:

$$
\begin{aligned}
& \|v A X+(1-v) X B\|_{2}^{2} \geq v^{2}\|A X-X B\|_{2}^{2}+r[\| X B \\
& \left.\left\|{ }_{2}^{2}+v\right\| A^{\frac{1}{2}} X B^{\frac{1}{2}}\left\|_{2}^{2}-2 \sqrt{v}\right\| A^{\frac{1}{4}} X B^{\frac{3}{4}} \|_{2}^{2}\right] \\
& +K r^{\prime} v^{2 v}\left\|A^{v} X B^{1-v}\right\|_{2}^{2}+2 v(1-v)\left\|A^{\frac{1}{2}} X B^{\frac{1}{2}}\right\|_{2}^{2}
\end{aligned}
$$

where, $K=\min \left\{K\left(\sqrt{\frac{v \lambda_{i}}{\mu_{j}}, 2}\right), 1 \leq i, j \leq n\right\}, r=\min \{2 v, 1-2 v\}$ and $r^{\prime}=\min \{2 r, 1-2 r\}$.

(ii) If $\frac{1}{2} \leq v \leq 1$, then:

$$
\begin{gathered}
\|v A X+(1-v) X B\|_{2}^{2} \geq(1-v)^{2}\|A X-X B\|_{2}^{2}+r[\| \\
A X\left\|_{2}^{2}+(1-v)\right\| A^{\frac{1}{2}} X B^{\frac{1}{2}} \|_{2}^{2}- \\
\left.\left.2 \sqrt{1-v}\left\|A^{\frac{3}{4}} X B^{\frac{1}{4}}\right\| \frac{2}{2}\right)\right]+K^{r^{\prime}}(1-v)^{2(1-v)} \| \\
A^{v} X B^{1-v}\left\|_{2}^{2}+2 v(1-v)\right\| A^{\frac{1}{2}} X B^{\frac{1}{2}} \|_{2}^{2}
\end{gathered}
$$

where,

$$
K=\min \left\{K\left(\sqrt{\frac{\lambda_{i}}{(1-v) \mu_{j}}, 2}\right), 1 \leq i, j \leq n\right\},
$$

$r=\min \{2 v-1,2(1-v)\}$ and $r^{\prime}=\min \{2 r, 1-2 r\}$.

Proof: To prove assertions of Theorem 3.1, we need to obtain assertions $\quad v A X+(1-v) X B, A X-X B, A^{\frac{1}{2}} X B^{\frac{1}{2}}$ and $A^{v} X B^{1-v}$. It is well known, that every positive semidefinite matrix is unitarily diagonalizable. Therefore, it concludes that there are unitary matrices $U, V \in M_{n}$ so that $A=U D U^{*}$ and $B=V E V^{*}$, where $D$ $=\operatorname{diag}\left(\lambda_{1}, \ldots, \lambda_{n}\right)$ and $E=\operatorname{diag}\left(\mu_{1}, \ldots, \lambda_{n}\right)$ with $\lambda_{i}, \mu_{i} \geq 0$ for $1 \leq i \leq n$.

Let $Y=U^{*} X V=\left[y_{i j}\right]$, then we have:

$$
\begin{aligned}
A^{v} X B^{1-v} & =\left(U D U^{*}\right)^{v} X\left(V E V^{*}\right)^{1-v} \\
& =U D^{v}\left(u U^{*} X V\right) E^{1-v} V^{*} \\
& =U\left(D^{v} Y E^{1-v}\right) V^{*}
\end{aligned}
$$

Thus, using unitarily invariant property of $\|.\|_{2}$, it follows that:

$$
\begin{aligned}
\left\|A^{v} X B^{1-v}\right\|_{2}^{2} & =\left\|U\left(D^{v} Y E^{1-v}\right) V^{*}\right\|_{2}^{2} \\
& \left.=\| D^{v} Y E^{1-v}\right) V^{*} \|_{2}^{2} \\
& =\sum_{i, j=1}^{n}\left(\lambda_{i}^{v} v_{j}^{1-v}\right)^{2}\left|y_{i j}\right|^{2}
\end{aligned}
$$

Analogously, we can prove that:

$$
\begin{aligned}
& v A X+(1-v) X B=U\left[\left(v \lambda_{i}+(1-v) \mu_{j}\right) y_{i j}\right] V^{*}, \\
& A X-X B=U\left[\left(\lambda_{i}-\mu_{j}\right) y_{i j}\right] V^{*}
\end{aligned}
$$

And:

$$
A^{\frac{1}{2}} X B^{\frac{1}{2}}=U\left[\left(\lambda_{i}^{\frac{1}{2}} \mu_{j}^{\frac{1}{2}}\right) y_{i j}\right] V^{*}
$$

We firstly suppose $0 \leq v \leq \frac{1}{2}$, then in view of the unitary invariance of the Hilbert-Schmidt norm, we have:

$$
\begin{aligned}
& \|v A X+(1-v) X B\|_{2}^{2} \\
& =\sum_{i, j=1}^{n}\left(v \lambda_{i}+(1-v) \mu_{j}\right)^{2}\left|y_{i j}\right|^{2} \\
& \geq v^{2} \sum_{i, j=1}^{n}\left(\lambda_{i}-\mu_{j}\right)^{2}\left|y_{i j}\right|^{2} \\
& +r \mu_{j}\left(\sqrt{\mu_{j}}-\sqrt{v \lambda_{i}}\right)^{2}\left|y_{i j}\right|^{2} \\
& +K r^{\prime} v^{2 v} \sum_{i, j=1}^{n}\left(\lambda_{i}^{v} \mu_{j}^{1-v}\right)^{2}\left|y_{i j}\right|^{2}+2 v(1-v) \\
& \sum_{i, j=1}^{n}\left(\lambda_{i}^{\frac{1}{2}} \mu_{j}^{\frac{1}{2}}\right)^{2}\left|y_{i j}\right|^{2} \\
& =v^{2}\|A X-X B\|_{2}^{2}+r\left[\|X B\|_{2}^{2}+v \|\right. \\
& \left.A^{\frac{1}{2}} X B^{\frac{1}{2}}\left\|_{2}^{2}-2 \sqrt{v}\right\| A^{\frac{1}{4}} X B^{\frac{3}{4}} \|_{2}^{2}\right] \\
& +K r^{\prime} v^{2 v}\left\|A^{V} X B^{1-V}\right\|_{2}^{2}+2 v(1-v)\left\|A^{\frac{1}{2}} X B^{\frac{1}{2}}\right\|_{2}^{2}
\end{aligned}
$$

i.e.,

$$
\begin{aligned}
& \|v A X+(1-v) X B\|_{2}^{2} \geq v^{2}\|A X-X B\|_{2}^{2}+r[\| \\
& \left.X B\left\|_{2}^{2}+v\right\| A^{\frac{1}{2}} X B^{\frac{1}{2}}\left\|_{2}^{2}-2 \sqrt{v}\right\| A^{\frac{1}{4}} X B^{\frac{3}{4}} \|_{2}^{2}\right] \\
& +K r^{\prime} v^{2 v}\left\|A^{V} X B^{1-V}\right\|_{2}^{2}+2 v(1-v)\left\|A^{\frac{1}{2}} X B^{\frac{1}{2}}\right\|_{2}^{2}
\end{aligned}
$$

This show (3.1) holds.

Using of unitarily invariant property $\|.\|_{2}$ and by (2:2); we can obtain (3.4).

This completes the proof.

Remark 2: Obviously, (3.3) and (3.4) are improvement of inequalities (3.1) and (3.2).

At the end, we recall the following Lemmas that are necessary to obtain the other inequalities by (2.1) and (2.2). 
Lemma 3.1. Bhatia (1996) (Cauchy-Schwarz inequality): Suppose $a_{i}, b_{i} \geq 0,(1 \leq i \leq n)$. Then:

$$
\sum_{i=1}^{n} a_{i} b_{i} \leq\left(\sum_{i=1}^{n} a_{i}^{2}\right)^{\frac{1}{2}}\left(\sum_{i=1}^{n} b_{i}^{2}\right)^{\frac{1}{2}}
$$

Lemma 3.2. Bhatia (1996) Let $A, B \in M_{n}$, then:

$$
\sum_{i=1}^{n} s_{j}(A B) \leq\left(\sum_{i=1}^{n} s_{j}(A) s_{j}(B)\right.
$$

Theorem 3.2. Let $A, B \in M_{n}$ so that $A$ and $B$ are positive semidefinite and $0 \leq v \leq 1$.

(i) If $0 \leq v \leq \frac{1}{2}$ then:

$$
v^{2}\|A\|_{2}^{2}+(1-v)^{2}\|B\|_{2}^{2} \geq v^{2}\left[\|A\|_{2}^{2}+\|B\|_{2}^{2}-2\|A B\|_{2}^{2}\right]
$$$$
+r\left[\|B\|_{2}^{2}+v\|A B\|_{1}-2 \sqrt{v} \sqrt{\|A\|_{1}} \sqrt{\left\|B^{3}\right\|_{1}}\right]
$$

$+K r^{\prime} v^{2 v}\left\|A^{v} B^{1-v}\right\|_{2}^{2}$

where, $K=\min \left\{k\left(\sqrt{\frac{v \lambda_{i}}{\mu_{j}}}, 2\right), 1 \leq i, j \leq n\right\}, r=\min \{2 v, 1-2 v\}$ and $r^{\prime}=\min \{2 r, 1-2 r\}$.

(ii) If $\frac{1}{2} \leq v \leq 1$, then we have:

$$
\begin{aligned}
& v^{2}\|A\|_{2}^{2}+(1-v)^{2}\|B\|_{2}^{2} \geq(1-v)^{2}\left[\|A\|_{2}^{2}+\|B\|_{2}^{2}-2\|A B\|_{1}\right] \\
& +r\left[\|A\|_{2}^{2}+(1-v)\|A B\|_{1}-2 \sqrt{1-v} \sqrt{\|B\|_{1}} \sqrt{\left\|A^{3}\right\|_{1}}\right] \\
& +K r^{\prime}(1-v)^{2(1-v)}\left\|A^{1-v} B^{v}\right\|_{2}^{2}
\end{aligned}
$$

where,

$K=\min \left\{k\left(\sqrt{\frac{\lambda_{i}}{(1-v) \mu_{j}}}, 2\right), 1 \leq i, j \leq n\right\}, r=\min \{2 v-1,2 v(1-v)\}$ and $r^{\prime}=\min \{2 r, 1-2 r\}$.

Proof: Here, to establish Theorem 3.2, we will first prove the assertion of Theorem 3.1 together with estimate 3.5 .

For $0 \leq v \leq \frac{1}{2}$, based on inequalities (2.1), (3.5) and (3.6), it follows that:

$$
\begin{aligned}
& \operatorname{tr}\left(v^{2} A^{2}+(1-v)^{2} B^{2}\right) \\
& =v^{2} \operatorname{tr} A^{2}+(1-v)^{2} \operatorname{tr} B^{2} \\
& =\sum_{j=1}^{n}\left(v^{2} s_{j}^{2}(A)+(1-v)^{2} s_{j}^{2}(B)\right)
\end{aligned}
$$

$$
\begin{aligned}
& \geq v^{2}\left[\sum_{j=1}^{n} s_{j}^{2}(A)+\sum_{j=1}^{n} s_{j}^{2}(B)-2 \sum_{j=1}^{n} s_{j}(A) s_{j}(B)\right] \\
& +r \sum_{j=1}^{n} s_{j}(B)\left[\sqrt{s_{j}(B)}-\sqrt{v s_{j}(A)}\right]^{2} \\
& +K\left(\sqrt{\frac{v s_{j}(A)}{s_{j}(B)}}, 2\right) r^{\prime} v^{2 v} \sum_{j=1}^{n}\left[s_{j}\left(A^{v}\right) s_{j}\left(B^{1-v}\right)\right]^{2} \\
& \geq v^{2}\left[\sum_{j=1}^{n} s_{j}^{2}(A)+\sum_{j=1}^{n} s_{j}^{2}(B)-2 \sum_{j=1}^{n} s_{j}(A B)\right] \\
& +r\left[\sum_{j=1}^{n} s_{j}^{2}(B)+v\right. \\
& \left.\sum_{j=1}^{n} s_{j}(A) s_{j}(A) s_{j}(B)-2 \sqrt{v}\left(\sum_{j=1}^{n} s_{j}^{\frac{1}{2}}(A) s_{j}^{\frac{3}{2}}(B)\right)\right] \\
& +K r^{\prime} v^{2 v} \sum_{j=1}^{n}\left[s_{j}\left(A^{v} B^{1-v}\right)\right] \\
& \geq v^{2}\left[\|A\|_{2}^{2}+\|B\|_{2}^{2}-2\|A B\| 1\right] \\
& +r[] B\left\|_{2}^{2}+v\right\| A B \|_{1}-2 \sqrt{v} \sqrt{\|A\|_{1}} \sqrt{\left\|B^{3}\right\|_{1}} \\
& +K r^{\prime} v^{2 v}\left\|A^{v} B^{1-v}\right\|_{2}^{2}
\end{aligned}
$$

It is trivial that:

$\operatorname{tr}\left(v^{2} A^{2}+(1-v)^{2} B^{2}\right)=v^{2} \operatorname{tr} A^{2}+(1-v)^{2} \operatorname{tr} B^{2}=v^{2}\|A\|_{2}^{2}+(1-v)^{2}\|B\|_{2}^{2}$

From two recent relations, it follows that:

$$
\begin{aligned}
& v^{2}\|A\|_{2}^{2}+(1-v)^{2}\|B\|_{2}^{2} \geq v^{2}\left[\|A\|_{2}^{2}+\|B\|_{2}^{2}-2\|A B\|_{1}\right] \\
& +r\left[\|B\|_{2}^{2}+v\|A B\|_{1}-2 \sqrt{v} \sqrt{\|A\|_{1}} \sqrt{\left\|B^{3}\right\|_{1}}\right]+K r^{\prime} v^{2 v}\left\|A^{v} B^{1-v}\right\|_{2}^{2}
\end{aligned}
$$

This estimate completes the proof of (3.7). The proof (3.8) is similar. So we omit its details. This completes the proof.

\section{Conclusion}

This paper obtained some refinements of Young type inequalities for scalars, then as applications of them, we presented some norm and trace inequalities.

\section{Acknowledgement}

The authors would like to thank the editor and the referees for their useful comments and remarks.

\section{Funding Information}

The research was supported by Lorestan University.

\section{Authors Contributions}

Both authors contributed equally and significantly in writing this article. Both authors read and approved the final manuscript. 


\section{Ethics}

Interested readers are encouraged to find the applications for the derived scalar inequalities. This is an interesting topic for future research.

\section{References}

Bhatia, R., 1996. Matrix Analysis. 1st Edn., SpringerVerlag, New York, ISBN-10: 0387948465, pp: 349.

$\mathrm{Hu}, \mathrm{X}$., 2012. Young type inequalities for matrices. J. East China Nor-Mal University, 4: 12-17.

Nasiri, L., W. Liao and M. Shakoori, 2016. A note on Young type inequalities. Submitted.

Wu, J.L. and J.G. Zhao, 2013. Operator inequalities and reverse inequalities related to the KittanehManasrah inequalities. Linear Multilinear Algebra, 62: 884-894. DOI: 10.1080/03081087.2013.794235

Zuo, H., G. Shi and M. Fujii, 2011. Refined young inequality with Kantorovich constant. J. Math. Inequal., 5: 551-556. 